\title{
Influence of electromagnetic (EM) waves polarization modes on surface plasmon resonance
}

\author{
Noor Faezah Murat ${ }^{1}$, Wan Maisarah Mukhtar $^{1 *}$, P Susthitha Menon ${ }^{2}$, Affa Rozana Abdul Rashid ${ }^{1}$, Karsono Ahmad \\ Dasuki $^{1}$ and Awangku Abdul Rahman Awangku Yussuf ${ }^{1}$ \\ ${ }^{1}$ Faculty of Science and Technology, Universiti Sains Islam Malaysia, 71800 Nilai Malaysia \\ ${ }^{2}$ Institute of Microengineering and Nanoelectronics (IMEN), Universiti Kebangsaan Malaysia, 43600 Bangi Malaysia
}

\begin{abstract}
Optical sensor based on surface plasmon resonance are widely used for various applications. In this paper, an optimization of EM waves polarization modes for the occurrence of SPR by using a modified optical waveguiding assembly has been discussed. A $633 \mathrm{~nm}$ of linearly polarized He-Ne laser was appointed as a light source. The polarization modes were controlled by rotating the position of $\mathrm{He}-\mathrm{Ne}$ laser. It was found that the optimum SPR signal with reflectance, $R=0.21$ a.u and SPR angle, $\theta_{\mathrm{SPR}}=48^{\circ}$ can be obtained as the position of laser was rotated at $90^{\circ}$, with the presence of polarizer. The location of the critical angle was observed at $\theta_{\mathrm{C}}=46^{\circ}$. For the validation purpose, a simulation based on Fresnel equation was conducted where the percentage difference between the experimental and theoretical results were analyzed. The percentage difference between experimental and theoretical results for both $\theta_{\mathrm{SPR}}$ and $\theta_{\mathrm{C}}$ were $9.09 \%$ and $15.00 \%$ respectively. In conclusion, an excellent agreement between the theoretical and experimental findings proved the stability of this SPR setup, where the EM waves polarization can easily be controlled by manipulating the position of laser.
\end{abstract}

\section{Introduction}

Surface plasmon resonance (SPR) technique has been used as one of the optical detection due to its powerful device for monitoring interactions in biological system [1]. The application also had been developed widely in the past two decades in areas such as environmental protection, biotechnology, chemical analytes, food safety and security $[2,3]$. Surface plasma wave (SPW) is an electromagnetic excitation existing on the boundary of a dielectric and a metal [4].

SPR is an optical method for measuring the refractive index of very thin layers of material adsorbed on a metal [5]. This can be concluded that SPR is a phenomenon that involving the interaction of matter and polarized light while surface plasmon polariton (SPP) is an excitation of photon in SPR. It can detect the small change of the refractive index on the metal surface by showing a sharp dip of curved which represent the minimum reflectance in SPR phenomenon [6]. The sharp dip of curve is called the SPR curve. It happens when ppolarized light strikes a thin-film metal under the condition of total internal reflection (TIR) [7].

The main objective of this research is to study the influence of EM waves polarization modes on SPR by controlling the position of linearly polarized $\mathrm{He}-\mathrm{Ne}$ laser. SPR experimental assembly consists of laser with wavelength of $633 \mathrm{~nm}$, a polarizer, a convex lens, a prism, and a rotational stage [8].

\section{SPR Phenomenon}

SPR phenomenon is a coupled electromagnetic field charge density oscillation that occurs on the boundary of metal-dielectric which have different sign (positive sign: dielectric, negative sign: metal) of dielectric constant as shown in Fig. 1 [9]. Among the numerous possible metals that can be used are silver and gold. Silver has the sharpest SPR resonance peak while gold shows excellent surface stability are commonly employed [10].

Other than that, the SPR phenomenon also take place when the wave vector of the incidence beam and the surface plasmon are equal and the reflected light intensity becomes completely attenuated. The promising potential of SPR sensors is that they are very high sensitivity to a change in the refractive index of the sensing medium [11].

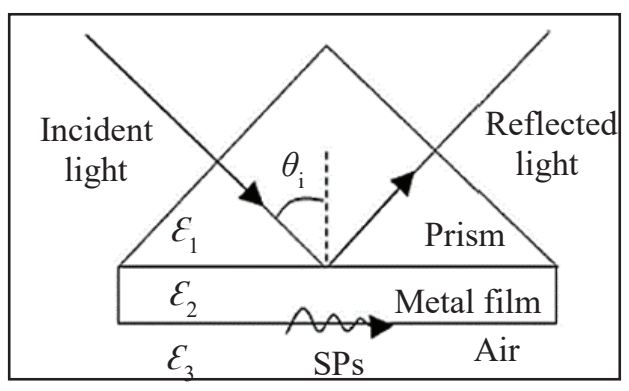

Fig. 1. SPR phenomenon [9].

\footnotetext{
* Corresponding author: wmaisarah@usim.edu.my
} 


\subsection{Maxwell Equation's}

Maxwell Equation's describes light interaction with matter. It's also used to show that in order for a point charge to produce electromagnetic waves, charge must accelerate. Maxwell's equations in a medium are given by [12]:

$$
\begin{gathered}
\nabla \cdot \vec{D}=\rho \\
\nabla \cdot \vec{B}=0 \\
\nabla \times \vec{E}=-\partial \vec{B} / \partial t \\
\nabla \times \vec{H}=J+\partial \vec{D} / \partial t
\end{gathered}
$$

where is $\vec{D}$ the dielectric displacement, $\rho$ is the density of charges, $\vec{B}$ is the magnetic field, $\vec{E}$ is the electric field, $\vec{H}$ is the magnetic field, and $\mathrm{J}$ is the current density. All the equations above represent Ampere's law, Faraday's law of induction, Gauss's law for electric and magnetic field respectively.

\subsection{Evanescent Wave}

Evanescent wave plays an important role in nano-optics field [13]. The evanescent wave does not transmit energy across the interface unless an absorbing or scattering species is presented in the lower index medium close to the boundary. This phenomenon happens when the wave strikes the medium at an angle greater than the critical angle, $\theta_{c}$ shown in Fig. 2 below $[14,15]$.

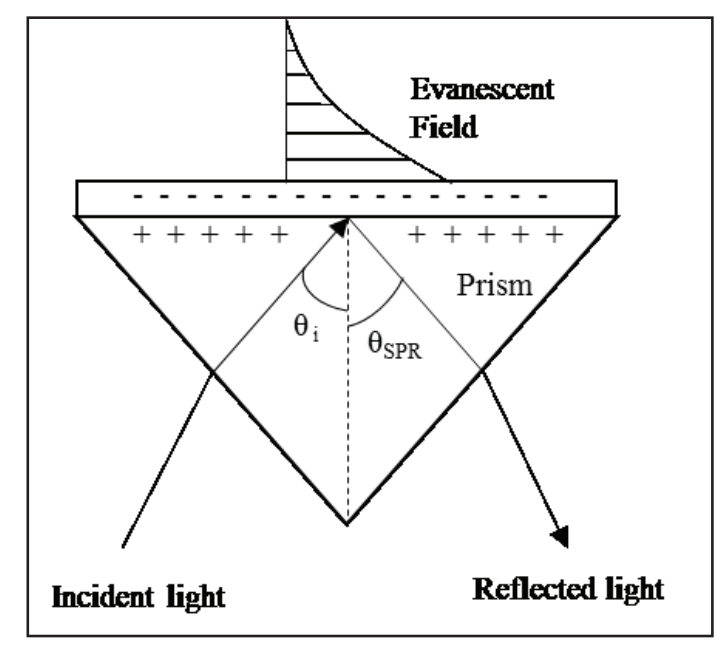

Fig. 2. The presence of evanescent field after light undergo total internal reflection [16].

\subsection{EM Polarization Mode}

Linear polarization can be classified into Transverse Magnetic mode (TM) or p-polarized and Transverse Electric mode (TE) or s-polarized with respect to the normal direction of the surface that is contained by the plane and the ray direction which is the propagation vector $[17,18]$. To recognize the polarization modes either s-polarized (horizontal polarization) or p-polarized (vertical polarization) value of reflectivity is observed. For s-polarized light, at first, the reflectivity increases gradually with the increment of incident angles. As the angles approaching $90^{\circ}$, the value of reflectivity increases more rapidly. Meanwhile, for the p-polarized light the reflectivity increases after it declines to zero [19].

It is crucial to understand the characteristic of light polarization in order to create SPR phenomena. EM waves polarization modes is one of the important requirements for SPP propagation to establish and influence the SPR phenomena. Since SPP can only be excited in p-polarization mode, the presence of Brewster angle, $\theta_{B}$ able to indicate type of polarization mode [20]:

$$
\theta_{B}=\tan ^{-1}\left(n_{2} / n_{1}\right)
$$

where $n_{1}$ and $n_{2}$ are refractive indexes of two different media.

At $\theta_{1}=0^{\circ}, \mathrm{p}$-polarization reflection is positive and has magnitude as pictured in Fig. 3. As $\theta_{1}$ increases, the magnitude decreases until it vanishes at the $\theta_{B}$. As $\theta_{1}$ increases beyond $\theta_{B}$, the reflection becomes negative and its magnitude increases until it reaches unity at the $\theta_{C}$. The wave undergoes TIR when $\theta_{l}$ is greater than $\theta_{C}$ [20].

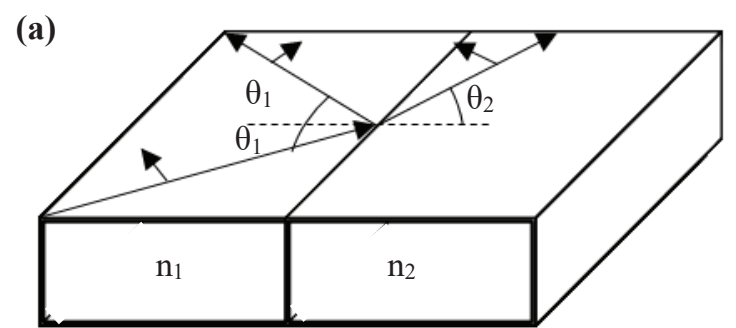

(b)

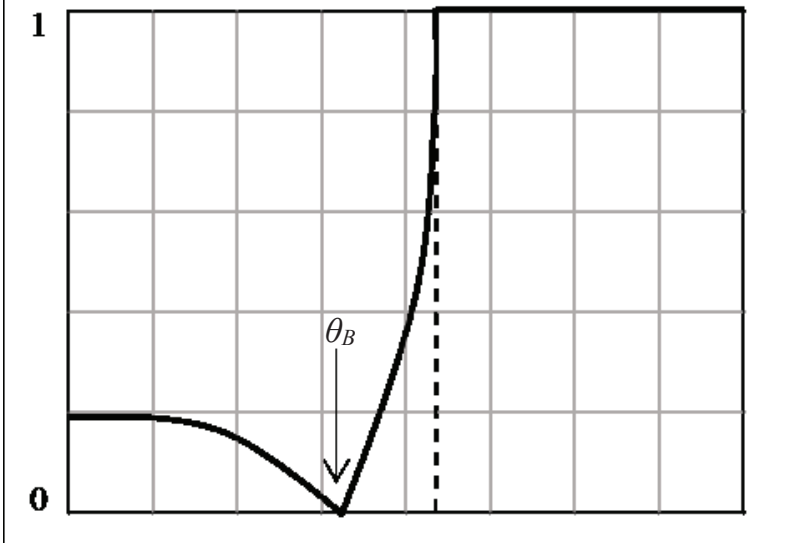

Fig. 3. (a) Magnitude of p-polarized waves for internal reflection (b) The magnitude vanished at the Brewster angle [20].

The power reflectance, $\mathfrak{R}$ and power transmittance, $T$ are defined as the ratio of power flow (along a direction normal to the boundary) of the reflected and transmitted waves to that incident wave. Since reflected and incident waves propagate in the same medium and make the same angle with normal to the surface, it follows that $\mathfrak{R}=|\mathrm{r}|^{2}$. The power reflectance at normal incidence for p-polarization at internal reflection is therefore [20]: 


$$
\mathfrak{R}=\left(\frac{n_{1}-n_{2}}{n_{1}+n_{2}}\right)^{2}
$$

\section{Methodology}

We started the experiment by building the SPR experimental setup. The setup consisted of few optical components as illustrated in Fig. 4. The linearly polarized $\mathrm{He}-\mathrm{Ne}$ laser with wavelength of $633 \mathrm{~nm}$ (Brand: Newport) was used for the light excitation. A polarizer (Brand: SIGMA KOKI) was placed between the laser and mirror with the distance of $18 \mathrm{~cm}$ and $7 \mathrm{~cm}$ respectively. The triangular prism (refractive index of 1.51) was positioned on top of mini labjack (Brand: Newport) which act as prism holder with distance of $26.5 \mathrm{~cm}$ from the mirror. The photodetector (Brand: Newport) was used to detect the reflected power in constant distance of $13 \mathrm{~cm}$ from the prism. The reading of the reflected power was measured by the power meter (Brand: Newport) which connected to the silicon photodetector.

Two types of prisms were employed in this experiment which were $50 \mathrm{~nm}$ gold coated prism and bare prism (without gold layer on top of the surface) as shown in Fig. $5[21,22]$. The function of bare prism is to determine the location of $\theta_{\mathrm{C}}$ and $\theta_{B}$. The presence of $\theta_{B}$ become an indicator of the propagation of p-polarized mode of the EM waves. Once the polarization modes were confirmed as p-polarized, the gold was deposited on the prism for the investigation of SPR signal.

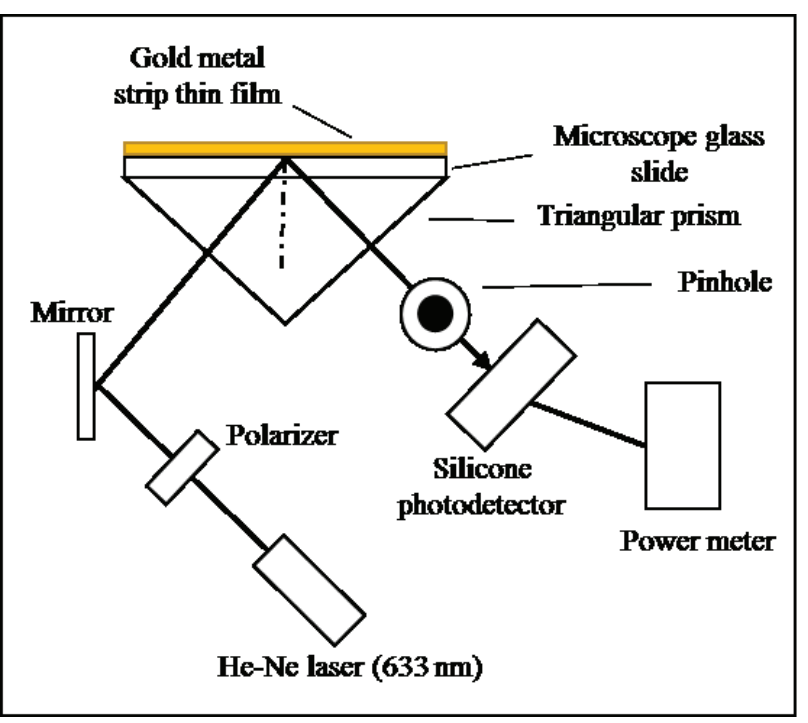

Fig. 4. Modified optical waveguiding assembly.

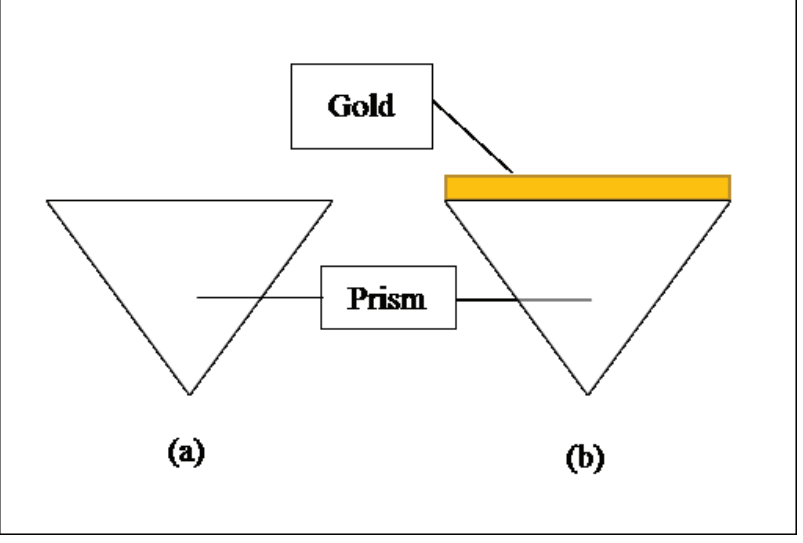

Fig. 5(a). Bare prism (b) gold-coated prism in Kretschmann configuration.

It is compulsory to excite p-polarization light as optical excitation of SPP since there are no solutions that exist for the case of s-polarization light [13]. In order to confirm that it was p-polarization light, the linearly polarized He-Ne laser source was rotated clockwise at three different angles such as at $0^{\circ} / 180^{\circ}, 90^{\circ} / 270^{\circ}$ and $45^{\circ}$ as illustrated in Fig. 6.

The next procedure was to perform an angular interrogation technique by rotating the prism in counter clockwise direction with an increment of $2^{\circ}$ for each reading. The range for the incident angles were between $20^{\circ}<\theta<60^{\circ}$ as illustrated in Fig. 7. The reading of power reflected was taken from power meter when photodetector was used to detect the reflected laser from the prism. The value of reflectance was calculated using the equation below:

$$
R(\text { a.u })=\frac{P_{\text {reflected }}(\mu W)}{P_{\text {incident }}(\mu W)}
$$

where $\mathrm{R}$ is the reflectance, $P_{\text {reflected }}$ is the reflected power and $P_{\text {incident }}$ is the incident power [8].

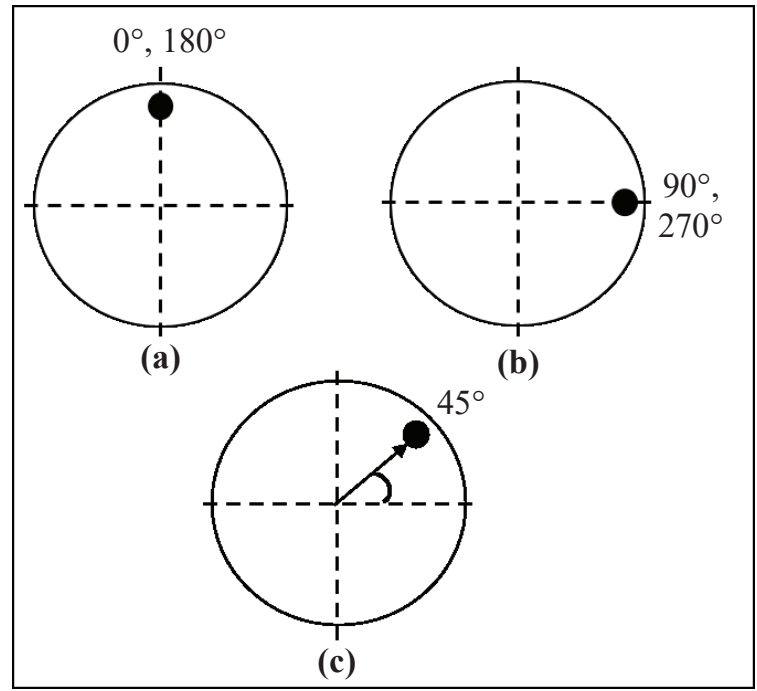

Fig. 6. Rotational angle of $\mathrm{He}-\mathrm{Ne}$ laser. 


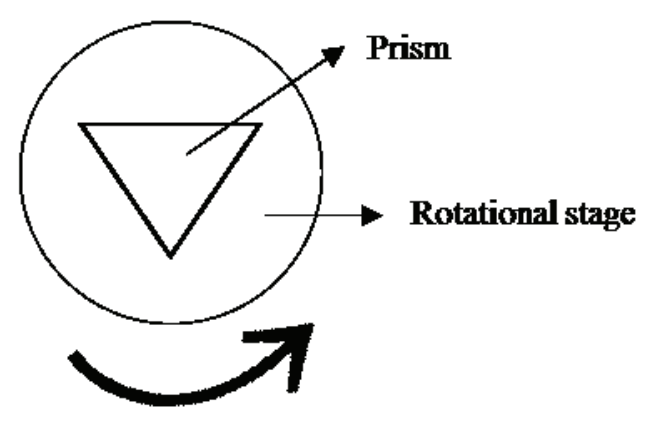

Rotate Counter Clockwise

Fig. 7. Rotating the prism in counter clockwise direction.

The quality of the SPR signal was examined by calculating the FWHM of the SPR curve, as follow [22, 23]:

$$
\Delta \theta=\theta_{2}-\theta_{1}
$$

where $\Delta \theta$ is FWHM, $\theta_{1}$ and $\theta_{2}$ are incident angle of SPR curve depth. As mentioned, FWHM represents the width of a reflection curve.

We performed a simulation by using WINSPALL 3.02 simulation developed by Knoll Group, Max-Plank Institute that based on a normalized Fresnel equation to compare the experimental and the calculated result. The results of the comparison represent the competency and accuracy of our SPR setup [22].

\section{Results and Discussions}

The occurrence of SPR can be observed as the position of laser was set at angle of $90^{\circ} / 270^{\circ}$ (Fig. 6 (b)). As the position of laser were varied into $0^{\circ} / 180^{\circ}$ (Fig. 6 (a)) and $45^{\circ}$ (Fig. 6 (c)), SPR signals were not observed. These results show that the p-polarized light can be obtained as the position of laser was at $90^{\circ} / 270^{\circ}$.

Fig. 8 shows the comparison between Brewster angle and critical angle for both experimental and simulation approaches. Due to the absence of a thin film layer, SPR cannot be observed thus results in no penetration of the surface plasmon waves evanescently [2]. The Brewster angle for simulation and experimental were $26^{\circ}$ and $30^{\circ}$ respectively with percentage difference of $15.38 \%$. The critical angle for simulation was $40^{\circ}$ and red shifted for experimental which was $46^{\circ}$ with percentage difference of $15 \%$. We expected that the location of SPR dips should be beyond these angles since the excitations of SPP must occur above the critical angles [24]. Table 1 summarizes the theoretical and experimental results for Brewster angle and critical angle of simulation and experimental results.
Table 1. Values of Brewster angle and critical angle obtained by simulation and experimental approaches

\begin{tabular}{|c|c|c|c|}
\hline Angle & Simulation & Experimental & $\begin{array}{c}\text { Percentage } \\
\text { Difference } \\
(\%)\end{array}$ \\
\hline Brewster & $26^{\circ}$ & $30^{\circ}$ & 15.38 \\
\hline Critical & $40^{\circ}$ & $46^{\circ}$ & 15.00 \\
\hline
\end{tabular}

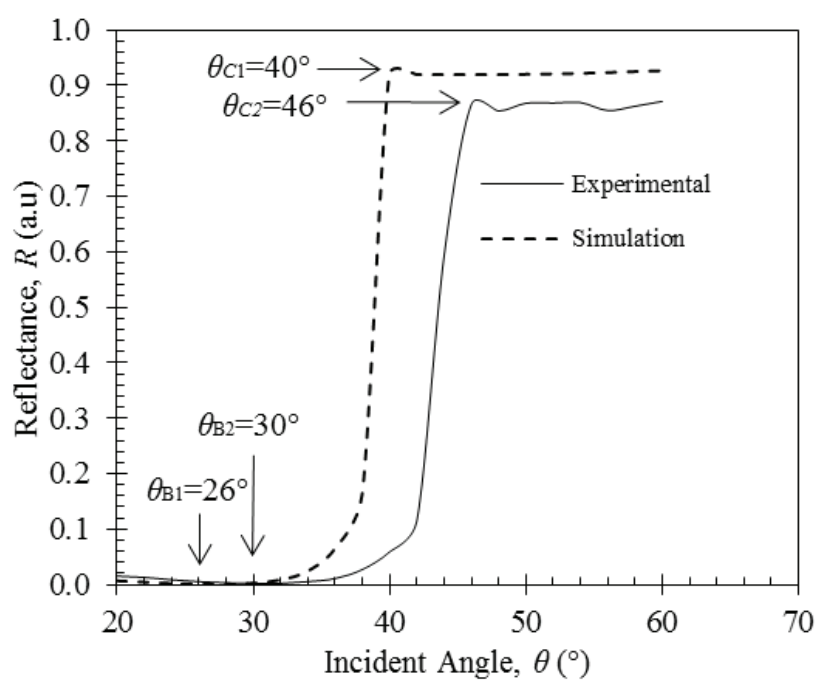

Fig. 8. Location of Brewster angle and critical angle for experimental and simulation results.

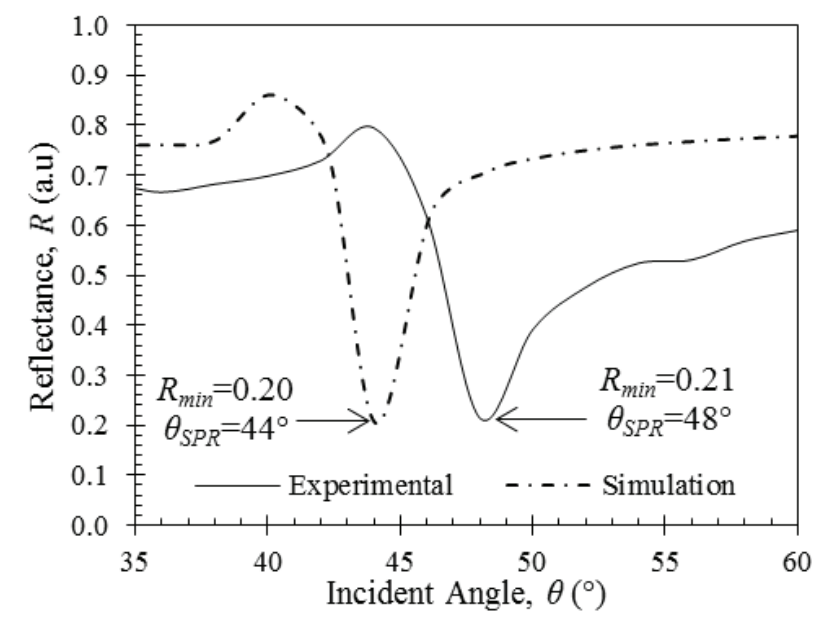

Fig. 9. Comparison of SPR curves of experimental and simulation results with the presence of gold thin layer deposited on the hypotenuse side of prism with thickness of $50 \mathrm{~nm}$.

Table 2. Summarization of SPR curve depth and FWHM

\begin{tabular}{|c|c|c|}
\hline Type & $\begin{array}{c}\text { SPR Curve } \\
\text { Depth, } \Delta \mathbf{R}(\mathbf{a . u})\end{array}$ & FWHM $\left(^{\circ}\right)$ \\
\hline Simulation & 0.66 & 2.1 \\
\hline Experimental & 0.59 & 2.0 \\
\hline
\end{tabular}


The SPR curves were analyzed by studying the graph of reflectance against incident angle as portrayed in Fig. 9. By comparing the experimental and simulation of SPR results, the optimum thickness of gold was obtained at $\mathrm{t}=50 \mathrm{~nm}$. It shows that the curve dip of experimental result was red shifted for about $4^{\circ}$ compared to simulation which is from $44^{\circ}$ to $48^{\circ}$. According to the simulation results, the SPR curve depth was obtained as $\Delta \mathrm{R}=0.66$ a.u while for experimental result $\Delta \mathrm{R}=0.59$ a.u with percentage difference of $10.60 \%$ was acquired. A good concurrent between simulation and experimental results had been proved based on FWHM analysis where the percentage difference between both of them were very small, about $4.7 \%$ as depicted in Table 2 .

\section{Conclusion}

In this study, the influence of EM waves polarization modes on SPR has been investigated theoretically and experimentally. We introduced a simple approach for the observation of SPR by controlling the position of $\mathrm{He}-\mathrm{Ne}$ laser. An excellent agreement between experimental and theoretical results indicate the validity and stability of our SPR setup.

The authors would like to acknowledge the support of Malaysian Ministry of Higher Education (MOHE) through Universiti Sains Islam Malaysia (USIM) under grant USIM/FRGS/FST/32/51514.

\section{References}

1. W. M. Mukhtar, S. Shaari, P. S. Menon, Adv. Sci Lett, 19, 66-64 (2013)

2. J. Homola, Anal. Bioanal. Chem., 377, 528-539, (2003)

3. Y. Chen, H. Ming, Photonic Sensors, 2, 37-49 (2012)

4. A. V. Zayats, I. I. Smolyaninov, A. A. Maradudin, Phys. Rep., 408, 131-314 (2005)

5. P. Pattnaik, Appl. Biochem. Biotechnol., 126, 79-92 (2005)

6. K. M. Byun, N.-H. Kim, J. W. Leem, J. S. Yu, Appl. Phys. B Lasers Opt., 107, 1-6 (2012)

7. A. Kausaite, M. Van Dijk, J. Castrop, A. Ramanaviciene, J. P. Baltrus, J. Acaite, A. Ramanavicius, Biochem. Mol. Biol. Educ., 35, 57-63 (2007)

8. W. M. Mukhtar, S. Shaari, P. S. Menon, Opto \& Adv. Mat., 7, 9-13 (2013)

9. Z. Yang, D. Gu, Y. Gao, Opt. Commun., 329, 180183 (2014)

10. B. Lee, S. Roh, J. Park, Opt. Fib. Tech., 15, 209-221 (2009)

11. S. Szunerits, N. Maalouli, E. Wijaya, J. P. Vilcot, R. Boukherroub, Anal. Bioanal. Chem., 405, 1435-1443 (2013)
12. H. D. Young, R. A. Freedman, University Physics with Modern Physics, 13, 1052 (2012)

13. L. Novotny, B. Hecht, Principles of Nano-Optics, Cambridge University Press, United Kingdom (2006)

14. T. Thio, Am. Sci. 94, 40-47 (2006)

15. P. L. Marston, Acoust. Soc. Am., 111, 2378 (2002)

16. Y. Tang, X. Zeng, J. Liang, Chem. Educ., 87, 7 (2010)

17. D. Halliday, R. Resnick, J. Walker, Fundamentals of Physics, 9, 1230-1233 (2010)

18. D. Sarid, W. Challener, Modern Introduction to Surface Plasmons: Theory, Mathematica Modelling and Applications, 1, 18-19 (2010)

19. W. A. A. Luhaybi, IUP, 12 (2014)

20. B. E. A. Saleh, M. C. Teich, Fundamentals of Photonics, 2, 212-214 (2007)

21. W. M. Mukhtar, S. Shaari, A. A. Ehsan, P. S. Menon, Opt Mat Exp, 4, 424 (2014)

22. N. F. Murat, W. M. Mukhtar, A. R. A. Rashid, K. A. Dasuki, A. A. R. A. Yussuf, IEEE-ICSE2016 Proc. 2016, 2, 244-247 (2016)

23. S. Y. Wu, H. P. Ho, Proc. 2002 IEEE Hong Kong Electron Devices Meet, 63-68 (2002)

24. W. M. Mukhtar, P. S. Menon, S. Shaari, M. Z. A. Malek, A. M. Abdullah, J. Phys. Conf. Ser., 431, 12028 (2013) 